\title{
THE INFLUENCE OF LIQUEFIED NATURAL GAS COMPOSITION ON ITS BEHAVIOR AS A COOLANT
}

\author{
A. Urbano and F. Nasuti \\ University of Rome "La Sapienza" \\ Dipartimento di Ingegneria Meccanica e Aerospaziale \\ 18 Via Eudossiana, Roma 00184, Italy
}

\begin{abstract}
Liquefied Natural Gas (LNG) is a suitable propellant to be used, together with liquid oxygen as oxidizer, in a liquid rocket engine, because of possible advantages with respect to hydrogen in specific applications. Often approximated as pure methane, LNG is a mixture of methane, other heavier hydrocarbons and nitrogen. If LNG is to be used in a regeneratively cooled liquid rocket engine, the knowledge of the thermodynamic and heat transfer characteristics when it flows in the cooling channels is of primary importance. The aim of the present work is to understand how the composition of LNG can influence the flow in the cooling channels. A parametric study is carried out considering different LNG compositions and heat flux levels. Attention is devoted to the pressure drop and cooling capabilities, which are the aspects that have to be controlled in a regenerative cooling system.
\end{abstract}

\section{INTRODUCTION}

In the recent years, an interest toward methane as a rocket engine fuel (together with oxygen as oxidizer) has been renewed because of several advantages it presents if compared with other commonly used fuels. If compared to hydrogen, methane is more dense, less expensive, and less cryogenic [1]. Moreover, methane is more energetic and has a higher coking temperature than all other hydrocarbons $[2,3]$. These advantages make this fuel interesting although it gives lower specific impulse than hydrogen, and is cryogenic and less dense compared to earth storable hydrocarbons. In nature, the major source of methane is the natural gas, which is in fact a gas mixture with a composition varying with extraction location. Raw natural gas is composed by more than $80 \%$ mole

This is an Open Access article distributed under the terms of the Creative Commons Attribution License 2.0, which permits unrestricted use, distribution, and reproduction in any medium, provided the original work is properly cited. 
fraction of methane, and for the remaining part by heavier hydrocarbons (primarily ethane, propane), nitrogen, water, carbon dioxide and elemental sulfur. The liquefaction process of natural gas requires removal of carbon dioxide, water, oxygen and sulfur compounds to prevent them from forming solids when the gas is cooled. As a result commercial LNG is typically made up of $90 \%$ or more of methane and of ethane, propane, butane and nitrogen in different percentages for the remaining part.

Further processing of LNG allows one to increase methane molar fraction with the obvious increase of the propellant cost. In fact, the cost of the necessary production and handling technology that can provide the desired liquid methane composition up to get pure methane, must be taken into account $[4,5]$. The cheaper LNG has therefore been considered as a possible rocket engine fuel instead of pure methane [6-8]. In fact, due to the large amount of methane contained, LNG should have the same kind of advantages as methane. However, using LNG rather than methane may affect the behavior of the different subsystems in such a way to yield changes of overall rocket performance that have to be correctly predicted.

Among the different subsystems, the present study focuses on the regenerative cooling system, with the aim of understanding the influence of the LNG composition on the fuel cooling capabilities and thermodynamic behavior. To this goal an accurate thermodynamic description of LNG is important and therefore it has to be described as a gas mixture by appropriate thermodynamic models. Cooling channels typically have a rectangular cross section, they are curved, and the heating is asymmetric. However, a simpler geometry of two-dimensional (2D) axisymmetric straight channels is considered in the present study to focus the attention only on the influence of the thermophysical properties over the coolant behavior. The most important parameters to analyze cooling system performance are the pressure drop and the temperature increase along the channel and also their local behavior or rather their evolution along the channel. In particular, it is interesting to observe how the pressure drop and heat transfer characteristics in the cooling channels change with LNG composition. To pursue this objective a fast and suitable numerical solver is essential. While the motivation for choosing a fast solver in a parametric study is obvious, a suitable solver is necessary to manage the complex flow modeling and thermodynamic phenomena, which affect the heat transfer to turbulent supercritical fluids. The proposed approximation is to use Parabolized Navier-Stokes equations (PNS) $[9,10]$. The parabolization is obtained by neglecting viscous derivatives in the space-marching direction and by considering the streamwise pressure gradient as a source term evaluated on the basis of the overall momentum balance. The algorithm is based on a finite volume approach with a Godunov-type scheme, which uses a modified Roe's approximate Riemann solver for a fluid governed by a generic Equation of State (EoS). Using PNS is particularly suitable for parametric analysis with a negligible loss of precision in physical modeling 
with respect to full Navier-Stokes solvers. Appropriate EoS and transport property relations for mixtures of real fluids must be used. The Groupe Européen de Recherches Gazières (GERG) [11] model has been selected for the EoS, whereas the Extended Corresponding States (ECS) models [12, 13] have been used for the transport properties.

In the following, the numerical and thermophysical models are presented. Then numerical simulations on heated channel flows of different LNG mixtures are analyzed, for normal and deteriorated heat transfer modes. A parametric study varying the LNG composition is carried out to understand the role of the percentage of the LNG main components on the cooling system performance. Two heat flux levels are considered to carry out the investigation both in case of a normal heat transfer (low heat flux) and the deteriorated heat transfer (high heat flux), which are the two forced convective heat transfer modes that can occur when heating a supercritical channel flow [14-16].

\section{NUMERICAL MODEL}

Parametric analysis of channel flows can be successfully made resorting to the PNS equations which are an approximation of the complete set of Navier-Stokes equations. Such an approach is considered in the present study: in fact, parabolization assumptions can be made for the channel flows of the present investigation. The present in-house PNS solver developed by the authors has been validated against experimental data [10] in conditions similar to those that have to be analyzed. For the sake of completeness, an introduction of the numerical model is reported, whereas further details can be found in [10].

Considering a Cartesian reference system for a straight tube, if $x$ is the streamwise direction and $y, z$ are the transverse directions, the conservative form of the three-dimensional PNS equations is:

$$
\frac{\partial \mathbf{F}_{\mathbf{e}}}{\partial x}+\frac{\partial \mathbf{G}_{\mathbf{e}}}{\partial y}-\frac{\partial \mathbf{G}_{\mathbf{v}}}{\partial y}+\frac{\partial \mathbf{H}_{\mathbf{e}}}{\partial z}-\frac{\partial \mathbf{H}_{\mathbf{v}}}{\partial z}=\frac{\partial \mathbf{P}}{\partial x}+\mathbf{Q}
$$

where the subscripts $e$ and $v$ indicate the Eulerian and viscous flux vectors, respectively, after the PNS approximation has been applied. In particular, these equations have been obtained by neglecting the streamwise viscous flux vector and the $x$ derivatives in the transverse viscous flux vectors (in $\mathbf{G}_{\mathbf{v}}$ and $\mathbf{H}_{\mathbf{v}}$, all the $x$ derivatives have been neglected). Moreover, the streamwise pressure gradient is considered as a source term $\partial \mathbf{P} / \partial x$ and, therefore, pressure does not appear in the streamwise Eulerian flux $\mathbf{F}_{\mathbf{e}}$. A further source term vector $\mathbf{Q}$ has been emphasized in Eq. (1) for the sake of generality. The resulting expressions of the flux vectors are: 


$$
\begin{aligned}
& \mathbf{F}_{\mathbf{e}}=\left\{\begin{array}{c}
\rho u \\
\rho u^{2} \\
\rho u v \\
\rho u w \\
\rho u h_{0}
\end{array}\right\} ; \quad \mathbf{G}_{\mathbf{e}}=\left\{\begin{array}{c}
\rho v \\
\rho u v \\
\rho v^{2}+p \\
\rho v w \\
\rho v h_{0}
\end{array}\right\} ; \quad \mathbf{H}_{\mathbf{e}}=\left\{\begin{array}{c}
\rho w \\
\rho u w \\
\rho v w \\
\rho w^{2}+p \\
\rho w h_{0}
\end{array}\right\} ; \\
& \mathbf{G}_{\mathbf{v}}=\left\{\begin{array}{c}
0 \\
\mu \frac{\partial u}{\partial y} \\
\frac{2}{3} \mu\left(2 \frac{\partial v}{\partial y}-\frac{\partial w}{\partial z}\right) \\
\mu\left(\frac{\partial v}{\partial z}+\frac{\partial w}{\partial y}\right) \\
u G_{v, 2}+v G_{v, 3}+w G_{v, 4}+k \frac{\partial T}{\partial y}
\end{array}\right\} ; \\
& \mathbf{H}_{\mathbf{v}}=\left\{\begin{array}{c}
0 \\
\mu \frac{\partial u}{\partial z} \\
\mu\left(\frac{\partial v}{\partial z}+\frac{\partial w}{\partial y}\right) \\
2 \frac{2}{3} \mu\left(2 \frac{\partial w}{\partial z}-\frac{\partial v}{\partial y}\right) \\
u H_{v, 2}+v H_{v, 3}+w H_{v, 4}+k \frac{\partial T}{\partial z}
\end{array}\right\}
\end{aligned}
$$

where subscripts 2,3 , and 4 indicate the second, the third, and the fourth components of vectors, respectively.

Finally, the pressure source term is given by:

$$
\mathbf{P}=\left\{\begin{array}{c}
0 \\
-p \\
0 \\
0 \\
0
\end{array}\right\}
$$

The Reynolds averaged Navier-Stokes (RANS) approach is considered to take into account the turbulence. The closure is obtained by computation of eddy viscosity according to the one-equation model of Spalart-Allmaras [17], modified to take into account the PNS hypothesis [10]. The diffusion-convection equation for the turbulent viscosity is solved together with Eq. (1), in which the turbulent viscosity and conductivity are added to the molecular values. 
Considering that the system of equations is hyperbolic-parabolic in the streamwise direction, the numerical solution of Eq. (1) can be obtained using a space-marching method. The present space-marching method relies on a finite volume scheme in which the unknown $\mathbf{F}_{\mathbf{e}}$ fluxes are integrated in the $x$ direction with Euler explicit scheme once the $\mathbf{G}_{\mathbf{e}}, \mathbf{G}_{\mathbf{v}}, \mathbf{H}_{\mathbf{e}}$, and $\mathbf{H}_{\mathbf{v}}$ fluxes have been evaluated at the cell interfaces. The Riemann solver is a modified version of the Roe's approximate Riemann solver [18] for Eq. (1) with generic EoS:

$$
h=h(p, \rho) ; \quad T=T(p, \rho) ; \quad \ldots
$$

where $h$ is the enthalpy; $T$ is the temperature; $p$ is the pressure; and $\rho$ is the density.

To make this integration possible, the pressure source term has to be known. In fact, one of the critical aspects of the use of PNS approach in channel flows is how to compute the streamwise pressure gradient, which has to be evaluated at each integration step. The assumed closure in the present approach is that of evaluating the $\partial \mathbf{P} / \partial x$ term in Eq. (1) with the iteration process, which finds the value of $\partial \mathbf{P} / \partial x$ such that the conservation of the integral momentum equation at each integration step along the channel length is satisfied [10].

\section{MIXTURE MODELS}

\subsection{Equation of State}

The most recent and reliable EoS applicable to LNG is that presented in the GERG study [11], which has been built especially to describe natural gas mixtures. The GERG EoS is based on the Helmholtz free energy, which takes into account the real behavior of fluids with some kind of departure function from the perfect gas solution. In particular, the GERG EoS is based on the pure substance EoS for each considered mixture component and correlation equations for binary mixtures consisting of these components. Mixing rules are applied to the reduced Helmholtz free energy $a$ :

$$
a(\delta, \tau, \bar{x})=\frac{A}{R T}=a^{0}(\rho, T, \bar{x})+a^{r}(\delta, \tau, \bar{x})
$$

with $\rho$ and $T$ being, respectively, the mixture density and temperature, $R$ being the gas constant, $A$ being the mass specific Helmholtz free energy, and $\bar{x}$ $=\left(x_{1}, x_{2}, \ldots, x_{N}\right)$ being the molar composition. The perfect gas term is given by the following expression:

$$
a^{0}(\rho, T, \bar{x})=\sum_{i=1}^{N} x_{i}\left[a_{i}^{0}(\rho, T)+\ln x_{i}\right]
$$


where the terms $a_{i}^{0}(\rho, T)$ are the Helmholtz free energy EoS for each of the considered species, for which explicit expressions can be found in [11]. On the other side, the residual term $a^{r}$ in Eq. (2), which corrects in the real fluid regime the perfect gas term $a^{0}$, includes both the departure Helmholtz free energy of each species $a_{i}^{r}$, whose expressions are given in [11] and which are linearly combined using the mole fraction $x_{i}$, and the residual behavior of the mixture (the double summation is called departure function):

$$
a^{r}(\delta, \tau, \bar{x})=\sum_{i=1}^{N} x_{i} a_{i}^{r}(\delta, \tau)+\sum_{i=1}^{N-1} \sum_{j=i+1}^{N} x_{i} x_{j} F_{i j} a_{i j}^{r}(\delta, \tau)
$$

where reduced mixture density $\delta$ and reduced mixture temperature $\tau$ are defined as

$$
\delta=\frac{\rho}{\rho_{r}(\bar{x})} ; \quad \tau=\frac{T_{r}(\bar{x})}{T} .
$$

The reducing parameters do not correspond with the mixture critical parameters, rather they are combinations of the critical parameters of the single species and are expressed as follows:

$$
\left.\begin{array}{rl}
\frac{1}{\rho_{r}(\bar{x})} & =\sum_{i=1}^{N} x_{i}^{2} \frac{1}{\rho_{c, i}} \\
& +\sum_{i=1}^{N-1} \sum_{j=i+1}^{N} 2 x_{i} x_{j} \beta_{\nu, i j} \gamma_{\nu, i j} \frac{x_{i}+x_{j}}{\beta_{\nu, i j}^{2} x_{i}+x_{j}} \cdot \frac{1}{8}\left(\frac{1}{\rho_{c, i}^{1 / 3}}+\frac{1}{\rho_{c, j}^{1 / 3}}\right)^{3} ; \\
T_{r}(\bar{x}) & =\sum_{i=1}^{N} x_{i}^{2} T_{c, i}+\sum_{i=1}^{N-1} \sum_{j=i+1}^{N} 2 x_{i} x_{j} \beta_{T, i j} \gamma_{T, i j} \frac{x_{i}+x_{j}}{\beta_{T, i j}^{2} x_{i}+x_{j}}\left(T_{c, i} T_{c, j}\right)^{0.5} .
\end{array}\right\}
$$

In Eqs. (5), $T_{c, i}$ and $\rho_{c, i}$ are the critical temperature and density of the $i$ th species, whereas $\beta_{\nu, i j}, \gamma_{\nu, i j}, \beta_{T, i j}$, and $\gamma_{\nu, i j}$ are the binary parameters relating the species $i$ and $j$. Further details and the values of coefficients included in Eqs. (2)-(5) can be found in [11]. The fundamental EoS describing the Helmholtz free energy as a function of temperature and density, with its derivatives, is sufficient to provide a complete description of the thermodynamic properties of the mixture. Once the EoS for the reduced Helmholtz free energy is available, the other thermodynamic properties of the mixture (the compressibility factor, the pressure, the enthalpy, specific heats, etc.) can be obtained from its derivatives with respect to reduced temperature and density. The range of validity of GERG EoS covers temperatures in the range $60 \leq T \leq 700 \mathrm{~K}$ and pressures lower than $70 \mathrm{MPa}$, with an uncertainty in density lower than $0.5 \%$.

As a validation of the present EoS, the evolution of the specific heat at constant pressure $c_{p}$ with temperature, for different pressures, has been compared in Fig. $1 a$ with data obtained by REFPROP software of the National Institute of Technology (NIST) [19], for a specified LNG mixture, showing a very good agreement. 


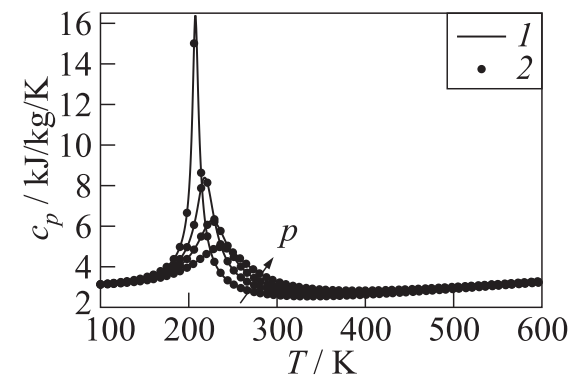

(a)

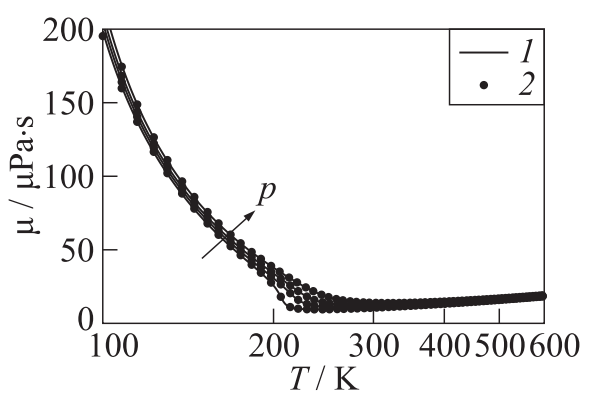

(b)

Figure 1 Comparison between present models (1) and NIST data (2) for an LNG mixture, for different pressures $(6,8,10$, and $13 \mathrm{MPa})$; LNG composition: $x_{\mathrm{CH}_{4}}=0.92$, $x_{\mathrm{C}_{2} \mathrm{H}_{6}}=0.04, x_{\mathrm{C}_{3} \mathrm{H}_{8}}=0.022$, and $x_{\mathrm{N}_{2}}=0.018:(a)$ specific heat at constant pressure; and $(b)$ dynamic viscosity

\subsection{Transport Properties}

The most commonly used approach followed in the literature to get viscosity and thermal conductivity of real fluid mixtures is the ECS theory. In the present study, transport properties have been evaluated according to ECS theory presented in $[12,13]$. Following this approach, viscosity and thermal conductivity are accurately evaluated for a reference fluid which is nitrogen $\left(\mathrm{N}_{2}\right)$, and whose properties are taken from [20]. The viscosity of a real fluid mixture is expressed assuming an excess property behavior [21]:

$$
\mu=\mu^{0}(T, \bar{x})+\mu^{r}(T, \rho, \bar{x})
$$

where $\mu^{0}$ is the diluted gas term and $\mu^{r}$ is the residual viscosity. The basic assumption of the ECS model is that

$$
\mu^{r}(T, \rho, \bar{x})=\mu_{0}^{r}\left(T_{0}, \rho_{0}\right) F_{\mu}^{r},
$$

namely, the residual viscosity of the mixture is equal to the residual viscosity of the reference fluid $\mu_{0}^{r}$ evaluated at the conformal temperature $T_{0}$ and density $\rho_{0}$ and modified for the correction factor $F_{\mu}^{r}$. Assuming that the residual viscosity of the reference fluid as a function of temperature and density is known, it is only necessary to evaluate conformal temperature and density and the reducing factor $F_{\mu}^{r}$. The conformal temperature and density are defined such that

$$
a^{r}(T, \rho, \bar{x})=a_{0}^{r}\left(T_{0}, \rho_{0}\right) ; \quad Z(T, \rho, \bar{x})=Z_{0}\left(T_{0}, \rho_{0}\right)
$$

where $a^{r}$ is the residual part of the reduced Helmholtz free energy $(a=A /(R T))$ and $Z$ is the compressibility factor of the mixture, whereas $a_{0}^{r}$ and $Z_{0}$ are the corresponding functions for the reference fluid. 
The evaluation of thermal conductivity follows the same procedure as for viscosity. In general the thermal conductivity of the fluid mixture will be expressed as $[22]$

$$
k=k^{0}(T, \bar{x})+k^{r}(T, \rho, \bar{x})+k^{c}(T, \rho, \bar{x})
$$

where $k^{0}$ is the diluted gas term; $k^{r}$ is the residual viscosity; and $k^{c}$ is the so-called critical enhancement which allows to predict the thermal conductivity peak in the vicinity of the critical point. The basic assumption of the ECS model is as in the case of viscosity:

$$
k^{r}(T, \rho, \bar{x})=k_{0}^{r}\left(T_{0}, \rho_{0}\right) F_{k}^{r},
$$

namely, the residual thermal conductivity of the mixture is equal to the residual thermal conductivity of the reference fluid $k_{0}^{r}$ evaluated at the conformal temperature $T_{0}$ and the density $\rho_{0}$ and modified for the correction factor $F_{k}^{r}$. Finally, the conductivity critical enhancement has been evaluated according to the model presented in [23].

For the dilute gas part, viscosity and thermal conductivity expressions for methane, ethane, and nitrogen are taken from [24], according to the empirical relations provided in $[25,26]$. The dependence of transport properties on temperature is as follows:

$$
\begin{aligned}
\mu_{i}^{0}(T) & =10^{-7} \exp \left[a_{\mu, 1, i} \log (T)+a_{\mu, 2, i} T^{-1}+a_{\mu, 3, i} T^{-2}+a_{\mu, 4, i}\right] \\
k_{i}^{0}(T) & =10^{-4} \exp \left[a_{k, 1, i} \log (T)+a_{k, 2, i} T^{-1}+a_{k, 3, i} T^{-2}+a_{k, 4, i}\right]
\end{aligned}
$$

where the coefficients $a_{\mu, i}$ and $a_{k, i}$ are taken from [26]. Once the data for the species are available, perfect gas mixture viscosity $\mu^{0}$ and thermal conductivity $k^{0}$ are computed according to [24] as

$$
\begin{aligned}
& \mu^{0}(T, \bar{x})=\sum_{i=1}^{N} x_{i} \mu_{i}^{0}\left(x_{i}+\sum_{\substack{i=1 \\
j \neq i}}^{N} x_{j} \phi_{i j}\right)^{-1} \\
& k^{0}(T, \bar{x})=\sum_{i=1}^{N} x_{i} k_{i}^{0}\left(x_{i}+\sum_{\substack{i=1 \\
j \neq i}}^{N} x_{j} \psi_{i j}\right)^{-1}
\end{aligned}
$$

where the coefficients $\phi_{i j}$ depend on the values of $\mu_{i}$ and $\mu_{j}$ and on the molar weights $W_{i}$ and $W_{j}$ of the species:

$$
\phi_{i j}=\frac{1}{2 \sqrt{2}}\left[1+\left(\frac{\mu_{i}}{\mu_{j}}\right)^{1 / 2}\left(\frac{W_{j}}{W_{i}}\right)^{1 / 4}\right]^{2}\left(1+\frac{W_{i}}{W_{j}}\right)^{-1 / 2}
$$


and the coefficients $\psi_{i j}$ necessary to evaluate the thermal conductivity are computed by a suitable correction of $\phi_{i j}$ :

$$
\psi_{i j}=\phi_{i j}\left[1+\frac{2.41\left(W_{i}-W_{j}\right)\left(W_{i}-0.142 W_{j}\right)}{\left(W_{i}+W_{j}\right)^{2}}\right] .
$$

The data for propane are not available in $[25,26]$. Hence, the dilute gas part of the viscosity model presented in [27] and of the thermal conductivity model presented in [28] have been considered to evaluate $\mu_{i, 0}$ and $k_{i, 0}$ for propane.

As a validation of the present transport property models, the evolution of the dynamic viscosity with the temperature, for different pressures, has been compared in Fig. $1 b$ with data obtained by REFPROP software of the NIST [19], for a specified LNG mixture, showing a very good agreement.

\section{TEST CASE PARAMETERS}

\subsection{Geometry and Boundary Conditions}

To investigate the influence of the LNG composition on its heat transfer properties, different numerical simulations have been carried out on a $2 \mathrm{D}$ axisymmetric geometry, that is a straight channel with a circular cross section of the diameter $D=4 \mathrm{~mm}$. In Fig. 2, a schematic of the test case with the enforced boundary conditions is shown: in the first 100 diameters no heat flux is applied so as to obtain a developed flow before heating in the last 200 diameters with a constant heat load of 5 or $7 \mathrm{MW} / \mathrm{m}^{2}$. For each heat flux level, the parametric study has been carried out keeping constant the following parameters: the inlet pressure $p_{\text {in }}=13 \mathrm{MPa}$; the inlet temperature $T_{\text {in }}=118 \mathrm{~K}$; and the mass flow rate per unit area $G=8500 \mathrm{~kg} / \mathrm{s} / \mathrm{m}^{2}$. The corresponding inlet Reynolds number, based

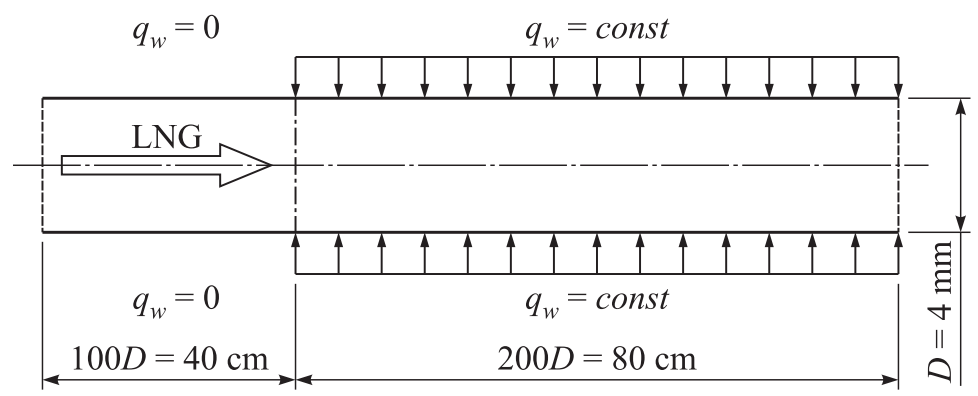

Figure 2 Schematic of the computational domain with the enforced boundary conditions (not to scale) 
on the diameter, is in the range $1.5 \cdot 10^{5}-3 \cdot 10^{5}$ and depends on the coolant composition. Both the geometry and the boundary conditions have been selected to get typical thermodynamic conditions of a rocket engine cooling channel. In particular, the work of Schuff et al. [1] has been used as a reference for this purpose. Note that the two heat flux levels have been chosen aiming to study the influence of LNG composition for the normal or deteriorated heat transfer [14-16].

The adopted mesh has 52 cells in the transverse direction, which are clustered towards the wall to describe also the viscous sublayer. In particular, the first cell to the wall has the height, in wall coordinates, smaller than one $\left(y^{+}<1\right)$.

\subsection{Liquified Natural Gas Compositions}

Table 1 The LNG composition range $[5,6,31-36]$

\begin{tabular}{lc}
\hline Species & Content \\
\hline $\mathrm{CH}_{4}$ & $80 \%-99 \%$ \\
$\mathrm{C}_{2} \mathrm{H}_{6}$ & $1 \%-17 \%$ \\
$\mathrm{C}_{3} \mathrm{H}_{8}$ & $0.1 \%-5 \%$ \\
$\mathrm{C}_{4} \mathrm{H}_{10}$ & $0.1 \%-2 \%$ \\
$\mathrm{C}_{5} \mathrm{H}_{12}$ and heavier & $\leq 1 \%$ \\
$\mathrm{~N}_{2}$ & $0 \%-5 \%$ \\
\hline
\end{tabular}

The LNG is natural gas that has been condensed to a liquid through a cooling process. Natural gas composition varies with its source (the location and the climate) and the processing history. Raw natural gas is a mixture of up to 21 components: methane $\left(\mathrm{CH}_{4}\right)$ is the primary component with a molar fraction of at least $80 \%$. Among the other components there are heavier hydrocarbons (ethane $\left(\mathrm{C}_{2} \mathrm{H}_{6}\right)$, propane $\left(\mathrm{C}_{3} \mathrm{H}_{8}\right)$, etc. $)$, carbon dioxide $\left(\mathrm{CO}_{2}\right)$, nitrogen $\left(\mathrm{N}_{2}\right)$, and water $\left(\mathrm{H}_{2} \mathrm{O}\right)[29,30]$. Before the liquefaction pro-

cess starts, hydrates, $\mathrm{CO}_{2}$, and other components that will freeze are removed. Also, hydrogen sulphide $\left(\mathrm{H}_{2} \mathrm{~S}\right)$ and mercury $(\mathrm{Hg})$ are removed to ensure that the gas is neither corrosive nor toxic [11]. The LNG composition will depend on the quality specification, and is quite variable around the world, but in general the main components molar fractions vary in the ranges reported in Table $1[5,6$, 31-36]: in the present study the heaviest hydrocarbon that has been considered is propane. Considering the ranges listed in Table 1, the compositions of Table 2 have been used for a first set of numerical simulations. The isobaric evolution with temperature of density, specific heat at constant pressure $c_{p}$, the dynamic viscosity $\mu$ and thermal conductivity $k$ are shown for the mixtures of Table 2 in Fig. 3 (these graphics have been obtained with the models presented in section 3 ). Also, pure methane curves have been reported for the sake of comparison. The temperature range goes from low subcritical to high supercritical values, whereas the inlet pressure is $13 \mathrm{MPa}$ which corresponds to a supercritical value for all the mixtures. From a qualitative point of view, the curves of Fig. 3 are similar to each other but in fact there are differences as large as $20 \%$. The purpose of the work is to investigate precisely how these differences could influence a heated channel flow. 
Table 2 Molar fractions of the species in different LNG mixtures $[5,6,31-36]$

\begin{tabular}{lcccc}
\hline Mixture & $\mathrm{CH}_{4}$ & $\mathrm{C}_{2} \mathrm{H}_{6}$ & $\mathrm{C}_{3} \mathrm{H}_{8}$ & $\mathrm{~N}_{2}$ \\
\hline MIX 1 & $92 \%$ & $4.0 \%$ & $2.2 \%$ & $1.8 \%$ \\
MIX 2 & $86 \%$ & $9.5 \%$ & $4.0 \%$ & $0.5 \%$ \\
MIX 3 & $93 \%$ & $5.0 \%$ & $1.5 \%$ & $0.5 \%$ \\
MIX 4 & $88 \%$ & $5.0 \%$ & $2.0 \%$ & $5.0 \%$ \\
\hline
\end{tabular}

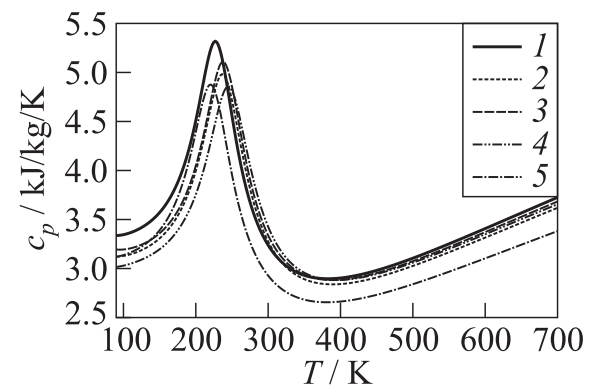

(a)

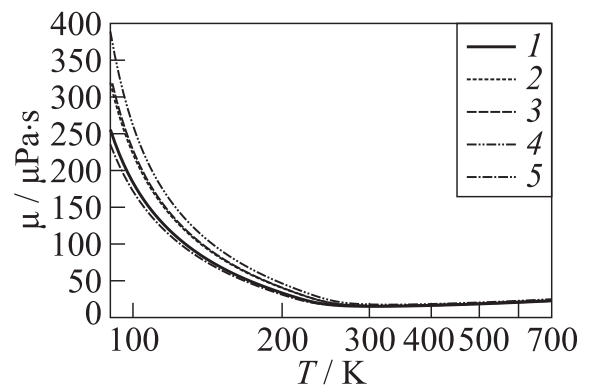

(b)

Figure 3 Specific heat at constant pressure $(a)$ and dynamic viscosity $(b)$ at the different LNG compositions of Table 2 for $p=13 \mathrm{MPa}: 1-\mathrm{CH}_{4} ; 2-$ MIX $1 ; 3-$ MIX 2; 4 - MIX 3; and 5- MIX 4

The mixtures of Table 2 are only representative of the typical LNG composition and do not give a complete overview. A better understanding of LNG behavior for varying composition can be obtained analyzing the influence of each species taken individually as the nonmethane part of the LNG mixture. To reach this goal, different binary mixtures $\mathrm{CH}_{4}-X$ are taken into consideration, where $X$ is either ethane, or propane, or nitrogen. For each of the three binary mixtures four compositions have been considered with methane molar fraction being $80 \%, 85 \%, 90 \%$, and $95 \%$.

\section{RESULTS}

\subsection{Liquefied Natural Gas Mixtures}

The results obtained for the heated channel flow considering LNG compositions of Table 2 are shown in Fig. 4 for both the investigated heat fluxes (5 and $7 \mathrm{MW} / \mathrm{m}^{2}$ ). Before analyzing the results, it has to be noted that the inlet bulk 
total pressure $p_{0}$ is slightly different for the different compositions: this is due to the fact that the static pressure, the static temperature, and the mass flow rate are the same for all the cases; hence, the inlet density and the inlet velocity differ and thus $p_{0}$. In particular, inlet velocity of pure methane is greater than that of the LNG mixtures which are characterized by a greater density.

The results are shown only for the heated part of the channel. In fact, the adiabatic part of the channel is needed only to obtain a developed flow before heating but the attention is focused on the heated part. Moreover, in the adiabatic part, being the supercritical fluid at very low temperature and thus similar to a liquid, the properties remain roughly constant.

The drop of the bulk total pressure with respect to the inlet total pressure is shown in Fig. 4a. For the higher heat flux, the pressure drops are greater as could be expected. On the other hand, for each heat flux the curves are similar for all the LNG compositions but the values are slightly different. The greater total drop is found for the methane case, whereas the drops for the LNG mixture cases are $5 \%$ to $10 \%$ smaller for both the heat fluxes. Reducing

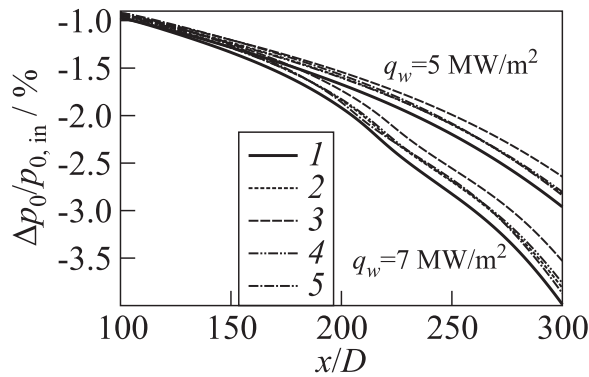

(a)

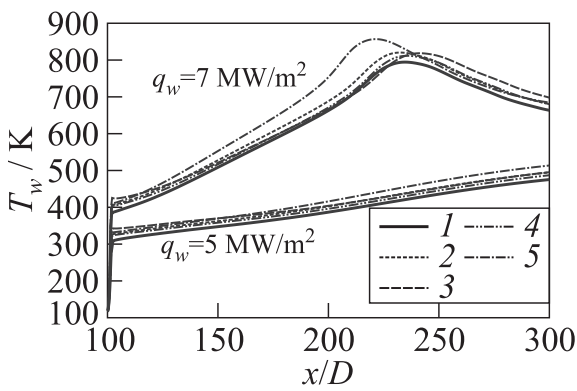

(c)

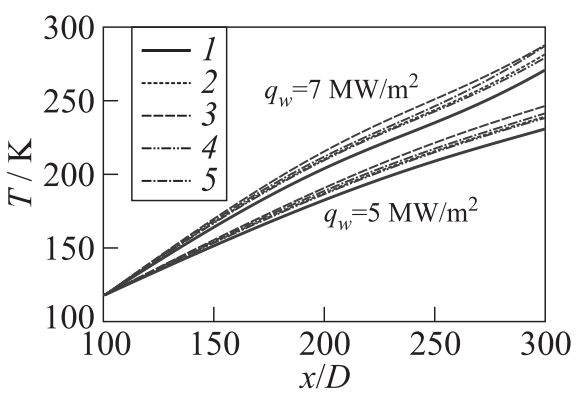

(b)

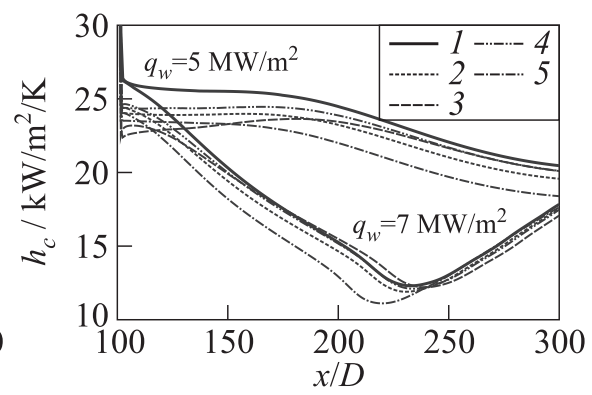

(d)

Figure 4 Heated channel flow with the different LNG compositions of Table 2 ( 1 $\mathrm{CH}_{4} ; 2-$ MIX 1; 3 - MIX 2; 4 - MIX 3; and 5-MIX 4): (a) bulk total pressure; $(b)$ bulk temperature; $(c)$ wall temperature; and $(d)$ convective heat transfer coefficient 
the pressure drops in the cooling channels is one of the main aspects in the design phase: for an assigned chamber pressure that has to be reached, the losses in the cooling channels influence the turbopump design and, hence, all the system. From this point of view, the lower pressure drops of the LNG mixtures compared to methane can be considered a positive property of LNG. Bulk and wall temperatures are reported in Figs. $4 b$ and $4 c$. In the adiabatic part not shown here, the temperatures can be considered constant. Moreover, the inlet temperature is one of the parameters set equal for all the test cases and for this reason all the temperature curves start from the same point in these figures. For the lowest heat flux, a normal heat transfer occurs. Due to the heat load, the total enthalpy increase in the heated part of channel results in an increase in both the static enthalpy (and, hence, the static temperature) and in the velocity. For a given heat flux, being all the test cases characterized by the same mass flow rate, the increase in the total enthalpy can also be considered as a fixed parameter of the analysis. As a consequence, different temperature increases can be related to the differences in the thermodynamic and transport properties. Among the investigated mixtures in Fig. 4, the smaller increase in the bulk temperature is found for methane and this is primarily due to its greater $c_{p}$ : bulk temperature for the LNG compositions are $3 \%$ to $7 \%$ greater. Also, the wall temperature is smaller for pure methane: at the channel exit, the LNG wall temperature is $4 \%$ to $8 \%$ greater than the methane one.

To describe the relation between $T, T_{w}$, and the heat transfer capabilities, the convective heat transfer coefficient $h_{c}$, defined as

$$
h_{c}=\frac{q_{w}}{T_{w}-T},
$$

is reported in Fig. 4d. This coefficient is useful to compare the heat transfer efficiency of different coolant flows. In fact, for an assigned temperature difference $\left(T_{w}-T\right)$, the greater $h_{c}$ the higher the heat flux towards the coolant flow, or for an assigned heat flux and bulk temperature, the higher $h_{c}$ the lower the corresponding $T_{w}$, which is a critical quantity that has to satisfy the cooling system constraints. The limit to the higher acceptable $T_{w}$ is set by either structural requirements or thermal cracking temperature, depending both on the used propellant and on the wall channel material features.

In the present discussion, when comparing different compositions for the same heat flux, $h_{c}$ is, in fact, the difference between the wall and the bulk temperatures. The $h_{c}$ curves of Fig. $4 d$ show that among the investigated compositions, the pure methane case has always the greater $h_{c}$ along the channel; it can be concluded that in the present conditions, the heat transfer efficiency decreases passing from pure methane to LNG. For the highest heat flux, a deterioration of the heat transfer occurs. In fact, the wall temperature has a peak which corresponds with a minimum in the heat transfer coefficient. From a quantitative point of view, the percentage differences between pure methane and the 
LNG mixtures are of the same order of magnitude as those observed for the normal heat transfer case $\left(q_{w}=5 \mathrm{MW} / \mathrm{m}^{2}\right)$. Namely, the differences are up to $8 \%$ for the temperatures. Despite that, it has to be considered that in the wall temperature peak region an increment of $8 \%$ could be important in terms of absolute values. As can be observed in Fig. $4 c$, the difference in the peak value of the wall temperature are up to $60 \mathrm{~K}$ between pure methane and LNG mixtures.

As a matter of fact, from the results presented in Fig. 4, it can be inferred that LNG composition influences both the pressure drop and the heat transfer behavior and this cannot be neglected in the design of a regenerative cooling system.

\subsection{Binary Mixtures: $\mathrm{CH}_{4}-\mathrm{N}_{2}, \mathrm{CH}_{4}-\mathrm{C}_{2} \mathrm{H}_{6}$, and $\mathrm{CH}_{4}-\mathrm{C}_{3} \mathrm{H}_{8}$}

As seen from the above results, only major differences between pure methane and LNG can be put in evidence and it is difficult to compare the LNG compositions to each other. On the contrary, the numerical simulations carried out with the binary mixtures presented in subsection 4.2 are useful to understand the role of each species in the LNG heat transfer behavior. The results of the binary mixtures are summarized in Fig. 5 for both the investigated heat fluxes: the graphics depict the total bulk pressure drop $\Delta p_{0}$ between the outlet and the inlet of the channel and the maximum wall temperature $T_{w, \max }$ reached along the channel as a function of the methane molar fraction in the binary mixture. A first comment to Fig. 5 is that nitrogen binary mixtures behave differently than the others for both the heat flux levels: this could be expected because of the difference in the thermophysical properties $\left(c_{p}, \mu, k\right)$ of nitrogen if compared

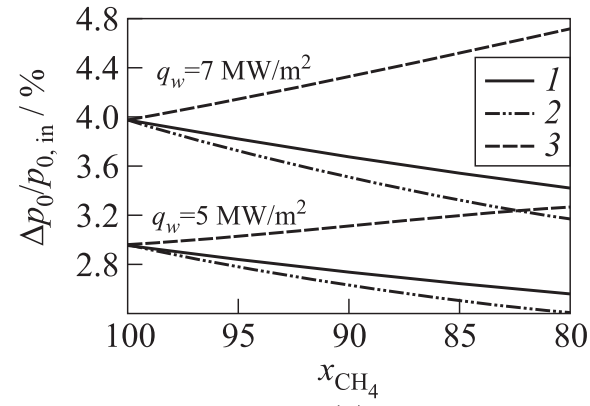

(a)

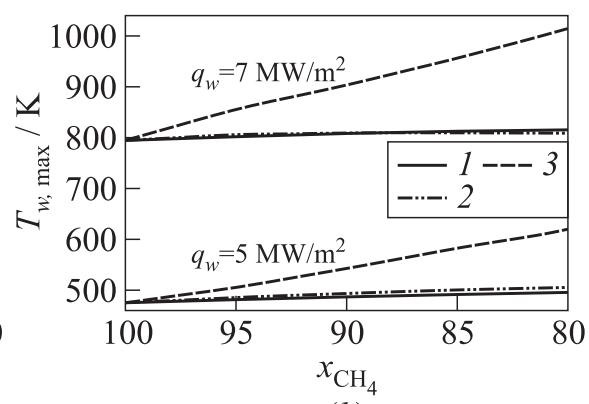

(b)

Figure 5 Total bulk pressure drop $(a)$ and maximum wall temperature $(b)$ for three different binary mixtures $\mathrm{CH}_{4}-X$ with $x_{\mathrm{CH}_{4}}$ methane percentage molar fraction: $1-$ $\mathrm{C}_{2} \mathrm{H}_{6} ; 2-\mathrm{C}_{3} \mathrm{H}_{8}$; and $3-\mathrm{N}_{2}$ 


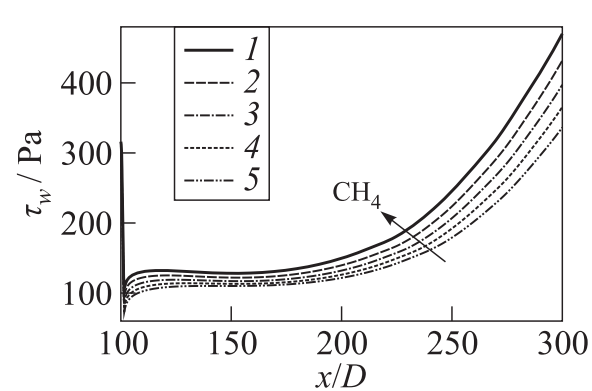

(a)

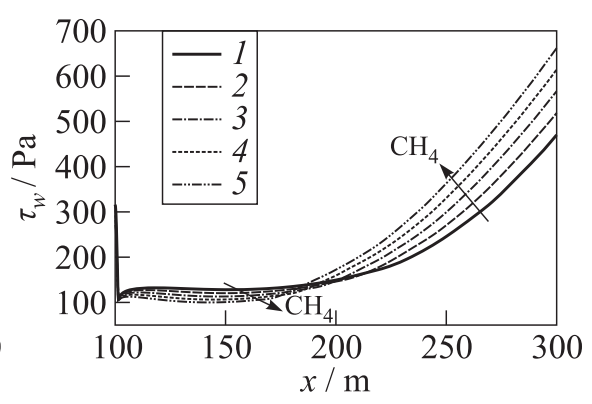

(b)

Figure 6 Evolution along the channel length of the wall shear stress for $\mathrm{CH}_{4}-\mathrm{C}_{3} \mathrm{H}_{8}(a)$ and $\mathrm{CH}_{4}-\mathrm{N}_{2}(b)$ binary mixtures, for $q_{w}=7 \mathrm{MW} / \mathrm{m}^{2}: 1-100 \% \mathrm{CH}_{4} ; 2-95 \% \mathrm{CH}_{4}$; $3-90 \% \mathrm{CH}_{4} ; 4-85 \% \mathrm{CH}_{4} ;$ and $5-80 \% \mathrm{CH}_{4}$

with the other investigated species which are hydrocarbons similar to methane. The $\Delta p_{0}$ increases with the nitrogen molar fraction, whereas it decreases with ethane and propane molar fractions. To understand the different $\Delta p_{0}$ trends, it is useful to analyze the behavior of the wall shear stress $\tau_{w}$ shown in Fig. 6 for the $q_{w}=7 \mathrm{MW} / \mathrm{m}^{2}$ test case: for propane mixtures (this is true also for ethane mixtures), $\tau_{w}$ decreases with the methane molar fraction. On the other hand, a different situation is observed for the nitrogen binary mixtures in the last part of the heated channel, where $\tau_{w}$ is greater for the mixtures with more nitrogen. Finally, with regard to the total pressure drop, Fig. $5 a$ shows that for the same methane molar fraction, the binary mixtures with ethane give a $\Delta p_{0}$ closer to the pure methane than the other mixtures.

To analyze the heat transfer behavior, it is worthwhile to distinguish between the two heat flux levels investigated. As can be observed in Fig. 7, for all the binary mixtures, for $q_{w}=5 \mathrm{MW} / \mathrm{m}^{2}$, a normal heat transfer occurs, whereas for $q_{w}=7 \mathrm{MW} / \mathrm{m}^{2}$, the heat transfer is deteriorated. For the normal heat transfer case, the maximum wall temperature reported in Fig. $5 b$ is reached at the end of the channel and always increases moving away from pure methane for all the binary mixtures. Also, for the heat transfer characteristics, nitrogen mixtures behave differently from the others. Nevertheless, the influence of the nitrogen molar fraction is clearly greater: the wall temperature difference with respect to the pure methane is about $30 \%$ for the $20 \%$ nitrogen case, whereas it is only $4 \%$ and $6 \%$ for the $20 \%$ ethane and $20 \%$ propane cases, respectively. The fact that methane is more efficient as a coolant than the investigated mixtures is also illustrated in Fig. 8: for $q_{w}=5 \mathrm{MW} / \mathrm{m}^{2}, h_{c}$ of pure methane is the greatest all along the channel. Moreover, nitrogen mixtures show the lower cooling capabilities which explain the corresponding greater increase in the temperature if compared with ethane and propane mixtures (see Fig. $5 b$ ). 


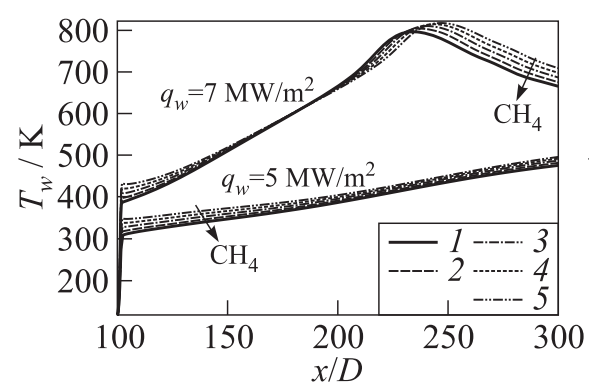

(a)

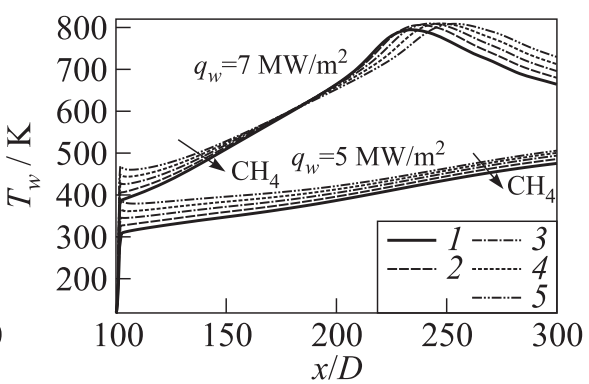

(b)

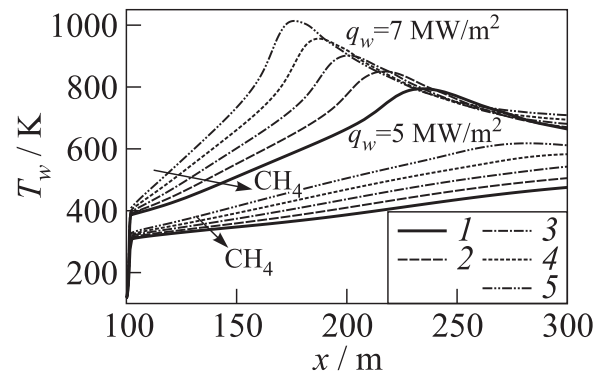

(c)

Figure 7 Evolution along the channel length of the wall temperature for $\mathrm{CH}_{4}-$ $\mathrm{C}_{2} \mathrm{H}_{6}(a), \mathrm{CH}_{4}-\mathrm{C}_{3} \mathrm{H}_{8}(b)$, and $\mathrm{CH}_{4}-\mathrm{N}_{2}(c)$ binary mixtures: $1-100 \% \mathrm{CH}_{4} ; 2-$ $95 \% \mathrm{CH}_{4} ; 3-90 \% \mathrm{CH}_{4} ; 4-85 \% \mathrm{CH}_{4}$; and $5-80 \% \mathrm{CH}_{4}$

For $q_{w}=7 \mathrm{MW} / \mathrm{m}^{2}$, the results shown in Figs. 7 and 8 give some information about the influence of the LNG composition over the heat transfer deterioration phenomenon. In particular, it is interesting to observe how the temperature peak position moves with the composition. Here again nitrogen influence is different from the propane and ethane influences. In fact, increasing of the nitrogen molar fraction moves the peak upstream and for the $20 \%$ nitrogen molar fraction case the deterioration occurs $60 D$ before than for the pure methane case. Conversely, increasing of propane and ethane moves the peak temperature downstream up to $12 \mathrm{D}$ and $20 \mathrm{D}$ for the $20 \%$ ethane and propane cases, respectively. This means that with nitrogen molar fraction increasing, a lower total heat load is sufficient for deterioration to occur, whereas increasing propane or ethane, a higher total heat load must be absorbed by the coolant for the phenomenon to occur. The percentage differences in the wall temperature are of the same order than those obtained for $q_{w}=5 \mathrm{MW} / \mathrm{m}^{2}$ except that the maximum wall temperature is reached when the wall temperature exhibits a peak. However, this brings to a wall temperature peak for the $20 \%$ nitrogen case which is $200 \mathrm{~K}$ greater than the pure methane one and reaches values as high as $1000 \mathrm{~K}$. 


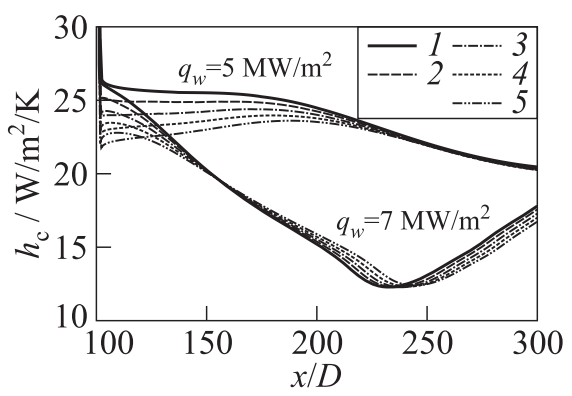

(a)

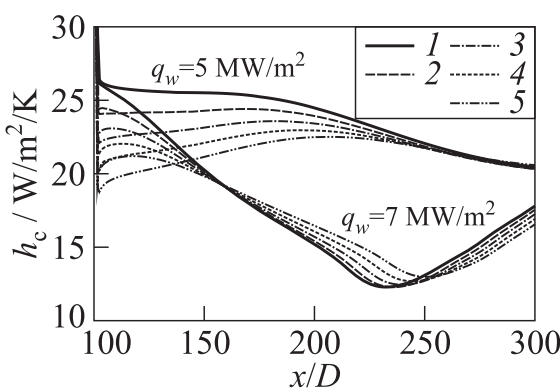

(b)

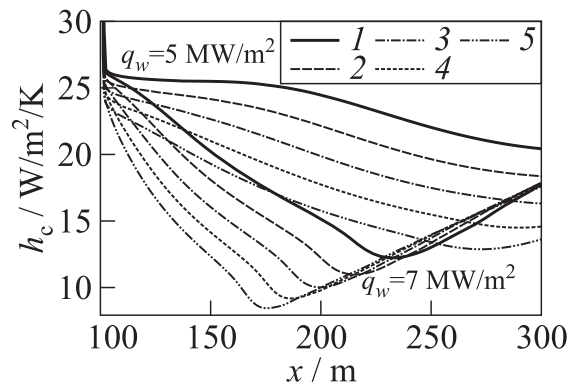

(c)

Figure 8 Evolution along the channel length of the heat transfer coefficient for $\mathrm{CH}_{4}-$ $\mathrm{C}_{2} \mathrm{H}_{6}(a), \mathrm{CH}_{4}-\mathrm{C}_{3} \mathrm{H}_{8}(b)$, and $\mathrm{CH}_{4}-\mathrm{N}_{2}(c)$ binary mixtures: $1-100 \% \mathrm{CH}_{4} ; 2-$ $95 \% \mathrm{CH}_{4} ; 3-90 \% \mathrm{CH}_{4} ; 4-85 \% \mathrm{CH}_{4} ;$ and $5-80 \% \mathrm{CH}_{4}$

On the contrary, the differences in the wall temperature peak values between ethane-methane and propane-methane mixtures are within $10 \mathrm{~K}$. Moreover, the increasing of the propane or ethane molar fraction seems to affect less and less the deterioration. The peak temperature even slightly decreases passing form the $15 \%$ to the $20 \%$ propane cases as can be observed in Fig. $5 b$. The same conclusions can be made observing the heat transfer coefficient in Fig. 8. For all the cases, the minimum $h_{c}$ takes place at the same abscissa as the wall temperature peak. It can be concluded that the increasing of the nitrogen molar fraction strongly reduces the $h_{c}$, whereas the presence of ethane and propane just slightly affects it, both from the qualitative and quantitative point of view.

\section{CONCLUDING REMARKS}

A parametric numerical study has been carried out to evaluate the influence of the LNG composition on the heat transfer behavior in the cooling channels of a 
regeneratively cooled liquid rocket engine. A suitable and fast numerical tool has been used for this purpose that is a PNS solver developed by the authors and validated against experimental data. Methane, ethane, propane and nitrogen have been considered as components of LNG mixtures for which transport and thermodynamic properties have been modeled using suitable mixture rules. The $2 \mathrm{D}$ axisymmetric test case of all the simulations was a straight channel with a circular cross section, uniformly heated. Comparisons have been made between different typical LNG compositions, and also between binary mixtures to investigate the effect of each species besides methane. Both normal and deteriorated heat transfer conditions have been taken into consideration. The bulk total pressure drop through the channel has been analyzed: ethane and propane diminish it, and propane more than ethane.

On the contrary, the increasing of the molar fraction of nitrogen causes an increase in the total pressure drop. Nevertheless, due to the low amount of nitrogen typically present in LNG and, hence, in the analyzed compositions in the present study, the total pressure drop is greater for methane than for LNG. The investigation on the cooling capabilities has put in evidence a great influence of nitrogen: the increasing of the nitrogen molar fraction causes a clear increase in the wall temperature both for the normal and deteriorated heat transfer cases. Also, the presence of ethane and propane slightly reduces the heat transfer capabilities of the coolant flow.

The study has also permitted to emphasize the influence of the LNG composition over the heat transfer deterioration phenomenon. In particular, it has been shown that, with the nitrogen molar fraction increasing, the temperature peak increases and moves upstream, whereas increasing the propane or ethane molar fraction the peak temperature moves downstream and eventually just slightly increases in value.

\section{ACKNOWLEDGMENTS}

The presented study on mixtures models has been carried out within the project "In-Space Propulsion 1" funded within the European Community Seventh Framework program, and coordinated in this part by Prof. M. Onofri.

\section{REFERENCES}

1. Schuff, R., M. Mayer, O. Sindiy, C. Ulrich, and S. Fugger. 2006. Integrated modeling and analysis for a LOx/methane expander cycle engine: Focusing on regenerative cooling jacket design. AIAA Paper No. 2006-4534. 
2. Cross, R.S. 1980. Combustion performance and heat transfer characterization of LOx/hydrocarbon type propellants. Report CR160874. Aerojet Liquid Rocket Company. Prepared for National Aeronautics and Space Administration.

3. Burkhardt, H., M. Sippel, A. Herbertz, and J. Klevanski. 2002. Comparative study of kerosene and methane propellant engine for reusable liquid booster stages. 4th Conference (International) on Launcher Technology "Space Launcher Liquid Propulsion." Liège.

4. Domaschenko, A. M., and Y. A. Kondrashkov. 2003. Technology of quality control of liquefied methane - a fuel for space rocket systems. J. Petroleum Sci. Eng. 39(11):656-61.

5. Domaschenko, A. M., A. L. Dovbish, R. V. Darbinyan, A. I. Lyapin, and V. A. Peredle'skii. 2004. Analysis of liquefied methane production technology depending on methane purity and production volume. J. Petroleum Sci. Eng. 40(3):145-48.

6. Han, P.-G., S.-W. Lee, K.-H. Kim, and Y. Yoon. 2004. Performance analysis of the thrust chamber in liquid rocket engine using liquefied natural gas as a fuel. AIAA Paper No. 2004-3860.

7. Higashino, K., M. Sugioka, T. Kobayashi, R. Minato, Y. Maru, Y. Sasayama, M. Otsuka, T. Makino, and H. Sakaguchi. 2008. Fundamental study on coking characteristics of LNG rocket engines. AIAA Paper No. 2008-4753.

8. Noguchi, Y., K. Taya, T. Hirai, A. Yui, and T. Makino. 2009. Conceptual design of a LOx/LNG rocket engine for a space tourism vehicle. AIAA Paper No. 2009-5138.

9. Urbano, A., and F. Nasuti. 2010. Numerical analysis of heated channel flows by a space-marching finite-volume technique. AIAA Paper No. 2010-4315.

10. Urbano, A., and F. Nasuti. 2011. Numerical analysis of heated channel flows by a space-marching finite-volume technique. J. Thermophys. Heat Transfer 25(2):28290.

11. Kunz, O., R. Klimeck, W. Wagner, and M. Jaeschke. 2007. The GERG-2004 widerange equation of state for natural gases and other mixtures. Technical Report. GERG TM15.

12. Klein, S. A., M. O. McLinden, and A. Laesecke. 1997. An improved extended corresponding states method for estimation of viscosity of pure refrigerants and mixtures. Int. J. Refrigeration 20(3):208-17.

13. Huber, M. L., A. Laesecke, and R. A. Perkins. 2003. Model for the viscosity and thermal conductivity of refrigerants, including a new correlation for the viscosity of r134a. Ind. Eng. Chem. Res. 42:3163-78.

14. Kelbaliev, R.F. 2001. Deterioration of heat transfer at supercritical pressures of a substance. J. Eng. Phys. Thermophys. 74:416-20.

15. Yamashita, T., H. Mori, S. Yoshida, and M. Ohno. 2003. Heat transfer and pressure drop of a supercritical pressure fluid flowing in a tube of small diameter. Memoirs of the Faculty of Engineering, Kyushu University 63(4):227-44.

16. Pioro, I.L., and R.B. Duffey. 2005. Experimental heat transfer in supercritical water flowing inside channels (survey). Nuclear Eng. Design 235(22):2407-30.

17. Spalart, P. R., and S. R. Allmaras. 1994. A one-equation turbulence model for aerodynamic flow. La Recherche Aerospatiale 1:5-21. 
18. Roe, P. L. 1981. Approximate Riemann solvers, parameter vectors, and difference schemes. J. Comput. Phys. 43:357-72.

19. NIST, National Institute of Standards and Technology. http://webbook.nist.gov/ chemistry/fluid/.

20. Lemmon, E. W., and R.T. Jacobsen. 2003. Viscosity and thermal conductivity equations for nitrogen, oxygen, argon, and air. Int. J. Thermophys. 25(1):21-69.

21. Huber, M. L., and J. F. Ely. 1992. Prediction of viscosity of refrigerants and refrigerant mixtures. Fluid Phase Equilibria 80:239-48.

22. Huber, M. L., D. J. Friend, and J.F. Ely. 1992. Prediction of thermal conductivity of refrigerants and refrigerant mixtures. Fluid Phase Equilibria 80:249-61.

23. Olchowy, G. A., and J. V. Sengers. 1989. A simplified representation for the thermal conductivity of fluids in the critical region. Int. J. Thermophys. 10:417-26.

24. McBride, B. J., and S. Gordon. 1994. Computer program for calculation of complex chemical equilibrium compositions and applications: I. Analysis. Cleveland, Ohio: National Aeronautics and Space Administration, Lewis Research Center. Reference Publication NASA RP-1311.

25. Boushehri, A., J. Bzowski, J. Kestin, and E. A. Mason. 1987. Equilibrium and transport properties of eleven polyatomic gases at low density. J. Phys. Chem. Ref. Data 16:445-67.

26. Svehla, R. A. 1995. Transport coefficients for the NASA Lewis chemical equilibrium program. NASA TM 4647.

27. Vogel, E., C. Kuchenmeister, and E. Bich. 1998. Reference correlation of the viscosity of propane. J. Phys. Chem. Ref. Data 27(5):947-55.

28. Marsh, K. N., R. A. Perkins, and M. L. V. Ramires. 2002. Measurement and correlation of the thermal conductivity of propane from $86 \mathrm{~K}$ to $600 \mathrm{~K}$ at pressures to 70 MPa. J. Chem. Eng. Data 47:932-40.

29. Farzaneh-Gord, M., A. Khamforoush, S. Hashemi, and H. P. Namin. 2010. Computing thermal properties of natural gas by utilizing aga8 equation of state. Int. J. Chem. Eng. Appl. 1(1):20-24.

30. NETL, National Energy Technology Laboratory. http://www.netl.doe.gov/.

31. Cook, R. T. 1984. Methane heat transfer investigation. NASA CR-171051.

32. Conrado, C., and V. Vesovic. 2000. The influence of chemical composition on vaporisation of LNG and LPG on unconfined water surfaces. Chem. Eng. Sci. 55:4549-62.

33. Nasrifar, Kh., and O. Bolland. 2006. Prediction of thermodynamic properties of natural gas mixtures using 10 equations of state including a new cubic two-constant equation of state. J. Petroleum Sci. Eng. 51:253-66.

34. Hissong, D. W. 2007. Keys to modeling LNG spills on water. J. Hazardous Mater. 140:465-77.

35. U.S. Department of Energy National Energy Technology Laboratory. 2007. LNG interchangeability/gas quality: Results of the National Energy Technology Laboratory's research for the FERC on natural gas quality and interchangeability. Report.

36. Querol, E., B. Gonzalez-Regueral, J. García-Torrent, and A. Ramos. 2011. Available power generation cycles to be coupled with the liquid natural gas (LNG) vaporization process in a Spanish LNG terminal. Appl. Energy 88:2382-90. 